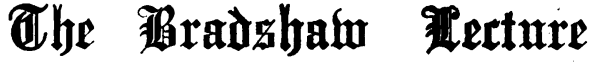

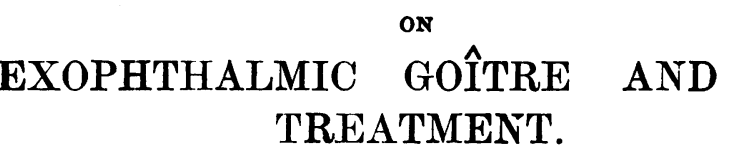

ITS

Delivered before the Royal College of Physicians of London.

By GEORGE R. MURRAY, M.A., M.D.CantaB., F.R.O.P., Health Professor of Comparative Pathology, University of Durham; Physician to the Royal Infirmary, Newcastle.

Mr. President and Gintlemen, - In the first place I must thank you very sincerely for the great honour which you have conferred upon me by your invitation to deliver the Bradshav lecture here to-day. When I read the list of distinguished physicians who have preceded me in this lectureship, I feel that my best efforts can only fall far behind the admirable contributions made by them to the science and art of medicine by means of this lecture. A few years ago, when I had the honour of delivering the Goulstonian lectures in this theatre, I selected the pathology of the thyrold gland as my subject, and devoted a considerable portion of one lecture to the pathology of exophthalmic goître. On this occasion I propose to consider the clinical symptoms and treatment of that interesting disease. This selection has been made because a considerable number of cases of this malady has come under my observation, of which I have tabulated 180 for the purpose of this lecture; 170 of these cases occurred in women and 10 in men, the frequency of the disease in the two sexes thus being in the proportion of 17 to 1 .

The Thyroid Grand.

Some enlargement of the thyroid gland is one of the most frequent aymptoms of exophthalmic goitre. The great frequency of this symptom is well shown by my series of 180 cases. In 172 of these the gland was enlarged at the time the patient was examined. In 5 others it was not enlarged at the time, but the history clearly showed that it had been so at an earlier stage of the disease. In only 3 cases there was no enlargement at the time of the examination, and no history of any previous enlargement could be obtained. So that in only $1 \frac{2}{3}$ per cent. of my cases was a goitre entirely absent. In a series of 26I cases collected by other observers 27, or rather more than ro per cent., had no goître. Mr. Arthur Maude, of Westerham, to whom I am greatly indebted for the generous loan of the MS. of an unpublished work on exophthalmic goitre, has found some enlargement of the thyroid gland at one time or another in all his cases. I am therefore inclined to believe that if all cases were observed frequently throughout the duration of the malady, some enlargement of the thyroid would be found in almost every one.

It is often difficult to ascertatn when the goitre has first appeared, as a moderate degree of enlargement may be attained before it attracts the notice of the patient or her friends; indeed, it is frequently discovered for the first time by the medical man, who looks for it in association with other eymptoms complained of. As a rule, no discomfort is occasioned by the goitre as long as the enlargement is slight or moderate in degree, so that it is not infrequently first noticed by the patient while looking at herself in a mirror, or a gradual tightening of the collar or neck-band leads to a careful examination of the front of the neck, when the swelling is discovered for the first time. Sometimes, however, a sense of fullness or constriction is felt in the neck, but such sensations naturally are more distinct when the enlargement is considerable. An enlargement of the thyroid gland is often the first sign of Graves's disease which is noticed. In a certain number of cases a simple parenchymatous goître is present for a considerable length of time before any symptoms of exophthalmic goître develop, as in at least fourteen of my cases. In two cases the goitre had been present for more than thirty years, and in other cases for periods of from three to twenty-five years, before the symptoms of Graves's disease began to develop.

As a rule, the enlargement of the thyroid gland is not very great. For the sake of convenience, the enlargement may be described as "slight" when it can be distinctly felt on palpating the gland, but is not always sufficient to attract the attention of the patient; as "moderate" when it is easily both seen and felt; and as "considerable" when it is obvious and disfiguring. In 51 cases the enlargement was slight, in 58 moderate, and in 29 considerable. In one the goitre was enormous. It had been present thirty-four years, and the circumference of the neck was 23 in. In 33 cases the size was not stated in the notes. In 8 cases there was no enlargement to be detected, though in 5 of these there had been a goitre at an earlier stage of the disease. As a rule, the enlargement of the gland is uniform, so that the natural shape is maintained. As the right lobe is larger than the left in the normal subject, we find the difference accentuated by the increase in size. In only 6 of my cases was the left lobe observed to be larger than the right. In a few cases adenomata or cyatic adenomate occur in the enlarged gland. In a few cases unilateral enlargement has been associated with homolateral exophthalmos. I have not, however, seen an example of this. The size of the goître may vary from week to week, or even from day to day. It may increase after unusual excitement or exertion, or during the menstrual period, to subside again in the course of a few days. The consistence of the gland waries somewhat in different cases, and in the same case at different times. As a rule, it is softer to the touch than the normal gland, but in cases of some duration it may become harder and smaller, owing to the development of fibrosis. The strong arterial pulsation in the carotid arteries, which is 80 frequently visible at the side of the neck, is not infrequently trangmitted to the goitre. The veins running over the surface are in some cases visibly dilated. A fine thrill can sometimes be felt when the finger is laid on the goitre, especially near the outer edge of each lateral lobe ; this was detected in 25 of $\mathrm{my}$ cases. A murmur may also be heard in many cuses over the goître, which may be either systolic in time, and therefore arterial, or continuous, and therefore venous in origin. In 63 of my cases a bruit was audible. In 41 of these it was systolic in time. In 19 there was a continuous venous murmur, which in to cases became much louder with each cardiac systole, or was accompanied by a superadded systolic murmur. In 31 cases no murmur was heard, and in the remaining 84 it was not noted.

Circulatory System.

In all cases of exophthalmic goître there is an increased frequency of the palse. At first palpitation may be only felt occasionally, dissppearing entirely between the attacks. Such attacks may be induced by unusual exertion. A characteristic feature of them, however, is that they may come on without any apparent reason when the patient is lying still in bed. In some cases the tachycardia comes on gradually, and may hardly attract the attention of the patient at all. In rare cases the onset is sudden, as in one of my cases in which it came on during the night in a girl who had previously been in good health, the other symptoms of Graves's disease appearing within a few days. The pulse may be increased to any rate between 90 and 200 beats a minute, according to the severity of the case. There is often a great variation in the frequency of the pulse at different stages of the disease, and also within short intervals of time in the same case, as slight exertion or emotion often greatly accelerates the pulse for a short time. For this reason it is difficult to give any average pulse-rate for each case. In considering my own cases I have for the purpose of comparison taken the highest observed pulse-rate in each case. This was recorded in 178 cases; in the other 2 it was increased, but the actual frequency was not noted.

$$
\begin{aligned}
& \text { Pulse Frequency. } \\
& \text { Between } 90 \text { and } \\
& \text { 100 , } 110 . . \\
& \text { 110 " } 120 \text {... } \\
& \begin{array}{lllll}
120 & 1, & 130 & \ldots
\end{array} \\
& \begin{array}{llll}
130 & 1,140 & 1 .
\end{array} \\
& \begin{array}{rrrr}
140 & 11 & 150 & \ldots
\end{array} \\
& \begin{array}{llll}
150 & , & 160 & \ldots \\
160 & \# & 170 & \ldots
\end{array} \\
& \begin{array}{rrrr}
160 & , & 170 & \ldots \\
170 & , & 180 & \ldots
\end{array} \\
& 180 \ldots, 190 \quad \ldots
\end{aligned}
$$

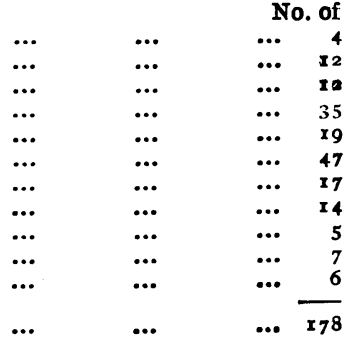

No. of Cases.

Thus in 101, or more than half the cases, the pulse was at times between 120 and 150 ; in 47 , or more than a quarter, it was between 140 and 150. The high frequence of 200 was observed during severe attacks of palpitation. In one such attack the pulse-rate fell from 180 to 140 in the course of a few minutes, and a quarter of an hour after the application of an icebag to the precordium it had fallen to 112 . The pulse, however, is usually regular, as irregularity was only observed in 12 of my cases. The action of the heart is similar to that which is produced in a healthy person by eevere 
exertion for a short period or by some violent emotion. On inspection diffuse pulsation all over the cardiac area is generally visible, extending as far to the left as the nippleline or beyond it. On palpation the impulse is felt over a larger area than usual. Taking the lowest and outermost point at which the apex beat could be distinctly felt in 93 of my cases, it was normal or internal to the left nipple-line in $1_{4}$, in 38 it was in the fifth left intercostal space in the nippleliue, in 34 it was in the same space but from $\frac{1}{2}$ in. to 2 in. begond the nipple-line. In 2 cayes it was in the sixth space in the nipple-line, and in 2 one fingerbreadth outside the nipple-line. In 3 cases it was in the anterior axillary line. In these cases with great dilatation of the left ventricle there is a danger of sudden fatal syncope, as one of these patients died suddenly during the night.

The heart sounds are very loud and distinct, the first sound at the apex especially being shorter, sharper, and louder than in the normal heart. The accentuation of the first sound may be so great that it is audible outside the chest. In one of $\mathrm{my}$ cases the first sound could be heard a yard away from the chest by the unaided ear and in another at a distance of aeveral inches. In the majority of early cases the sounds are clear and no murmur is audible. In the later stages, however, murmurs are not uncommon. A loud rough systolic murmur is frequently heard over the inner end of the second left intercostal space. This murmur alone was present in 26 of my cases, in some of which it was almost purring in character. In 19 cases a systolic murmur was heard at the apex only. while in $18 \mathrm{cases}$ a murmur was heard both at the apex and over the pulmonary area as well. In 45 no murmur was heard, and in the remainder it was not noted. The character of these murmurs indicates that they are usually functional in origin and they resemble haemic murmurs, al though they occur in many cases in which there is no distinct anaemia. True valvular lesions may occur in cases of exophthalmic goître, but should be regarded as complicatione, as they are usually the result of antecedent rheumatism or chorea.

Of vascular symptoms the most striking is the arterial throbbing which is most noticeable in the carotids. It is varlable in intensity, and in some cases is felt more especially in the temporal or cranial arteries. Strong pulsation may also be felt at times in the abdominal aorta and in the femoral arteries. The radial pulse, as we have already seen, varies greatly in frequency, bat is usually small and of low tension. Though the blood pressure was found to be normal by $P$. Marie in the two cases in which he measured it, haemorrhages from various parts of the body are occasional symptoms. Epistaxis is the most common, but haemoptysis, apart from pulmonary tuberculosis, haematemesis and bleeding from the skin have also been recorded.

\section{OCULAR SYMPTOMS}

Exophthalmos effects such a striking change in the appear ance of the patient that to many it is one of the most dis tressing features of the malady. Although so obvious when present, it is important to bear in mind that this sign is entirely absent in many cases. A note was made as to the condition of the eyes in 170 of $\mathrm{my}$ cases. In 120 exophthalmos was present; in 3 it had been present at an earlier stage but had disappeared before I saw them. In 47 cases, or more than 25 per cent., there was no exophthalmos at all. Exophthalınos generally develops slowly, but in some cases it comes on rapidly in the course of a few days. In cases in which it is present in a slight degree, it may rapidly increase if there is an acute exacerbation of the other symptoms. It is usually bilateral and equal in degree in both eyes, but in some cases it is unilateral, or more marked on one side than on the other. The exophthalmos may vary from a slight fullness of the eyeball, over which the eyelids can still meet easily, to such marked projection that the sclerotic above and below the margin of the cornea, and even the insertions of the recti muscles are visible, and the eyelids cannot be brought together at all. In marked cases the eyes remain open during sleep. In one of my cases, which I examined whlle asleep in the daytime, the eyes were directed forwards and the lower edge of the upper eyelids only descended as far as the upper margins of the pupils, which were of medium size though fully exposed to a strong direct light from windows facing south in the middle of the day in the month of August; in other cases the pupils were contracted. The amount of prominence of the eyeballs varies in the same case from time to time. Patients who have this gymptom in a marked form are very liable to suffer from attacks of conjunctivitis, owing to the increased exposure of the surface of the eyeball to the action of dust and bacteria floating in the air, wh ich is all the more apt to occur when the retraction of the upper eyelid with absence of blinking, known as Stellwag' sign, is present as well. This sign may be present when there is no exophthalmos, or it may precede its development. When it is present alone it imparts a somewhat staring or startled expression to the face. It was present in 70 of my cases, in 55 it was absent, and in an equal number it was not noted. This sign may vary in degree nr even disappear at times in the same case. It may on appear under the influence of a strong emotion, or when the eyes are suddenly turned in a new direction and disappear again at once. 'I'he delay in the normal descent of the upper eyelid when the eyes are directed in a downward direction which is so well known as von Graefe's sign, was present in 49 out of 141 of my cases in which it was specially looked for. It is to be remembered that this sign was observed by Dr. Sharkey in 14 out of 613 patients, none of whom suffired from Graves's disease. In one of my cases von Graefe's was the only ocular sign present. In 36 cases it was associated with exophthalmos and retraction of the npper eyelids, in 9 it occurred with exophthalmos but without stellwag's sign. In 19 cases in which both exophthalmos and Stellwag's sign were present von Graefe's sign was absent. Weakness of convergence, as described by Moebius, has been observed in some of my cases, but as a rnle I have not found it to be so marked as to be regarded as anything more than an indication of some slight weakness of the recti muscles. Paralysis of one or more of the external muscles of the eye has been described, especially by Mr. Maude, in a good many cases, the details of which are probably familiar to you. The paralysis may be partial or complete, bilateral or unilateral, and may involve a single muscle or all, causing complete external ophthalmoplegia, as in one of my own cases. The elevator of the upper lid usually escapes, though ptosis mav occur alone without ocular paralysis, as in cases recorded by Mannheim and by West. The pupils are usually normal in size and in reactions and vision is unaffected. Lesions of the cornea, such as have been described by von Basedow and von Graefe, were absent in my cases. The eyelids are swollen in some cases, the exact cause of this being uncertain. It has been described by some as a circumscribed oedema of one or both eyelids, but in the cases I have seen it has appeared to be more the result of looseness of the skin, possibly associated with relaxation of the orbicularis muscle. Pigmentation of the eyelids is a common symptom. A rapid tremor of the eyelids occurs in some cases when the eyes are shut, but disappears when they are opened.

\section{Nervods SYMPTOMS}

The mental condition in exophthalmic goître is peculiar, and in some cases this may be noticed even before the other signs of the malady become apparent. The mental state in its typical form is one of subdued excitement or nervousness, such as is seen in a person undergoing some trying ordeal. It may vary in different cases according to the natural temperament of the patient. Thus in some there is marked irritability of temper. and trifling circumstances which would have passed unheeded in health become sources of acute annoyance. An intolerance of strangers, due partly to a reluctance to meet them on account of the facial appearance produced by the exophthalmos, occurs in some cases, and associated with it, or even independently of it, there may be a sense of great discomfort when in a crowded building, such as a church or theatre. I have not noticed the abnormal love of dress and craving for entertainments which have been df scribed by Moebius. Some patients are very restless in movement. while others suffer from the peculiar restlessness of mind described by the late Sir J. Russell-Reynolds as a "chorea of 1deas." Some are always bright and optimistic, while others are discontented and melancholy. In spite, however, of this peculiar mental state, intellectual work of a high clase may be accomplished by those suffering from Graves's disease. Thus, one lady who was under my care during the greater part of a severe attack of exophthalmic goitre, which lasted some seven years before she recovered, occupied the time of her enforced leisure by acquiring a knowledge of the Tarkish language. In some cases there are definlte visual and auditory hallucinations. Careful inquiry is necessary to obtain a history of these hallucinations, as patients are often disinclined to mention their occurrence. In 10 of my cases, in which there was no insanity, definite hallucinations occurred. In one case a man had been killed by an accident in the 
district in which the patient lived, and some days after his burial the patient thought she saw him enter by the door. Another patient thinks she sees objects drop on to the floor from the chair, or the door open and on one occasion she heard a scrimmage in the yard and some one enter the house. In other cases figures or animals were more or less distinctly seen, especially on waking during the night. In one of these cases the faces of the figures were clearly recognized as being those of people who were dead, who appeared just as when they had last been seen. In acute fatal cases various mental symptoms occur near the end which resemble those of the delirium of fever. F. Müller has observed stupor, terror, hallucinations, and confusion in such cases. In the cases of this clasg which I have seen, the mental condition closely resembled that of a patient suffering from a severe attack of enteric fever or pneumonia with a mild form of delirium from which he could be roused to give rational answers to questions.

Actual insanity develops in a few cases of Graves's disease, and should perhaps be regarded more as a complication than as part of the disease. In the experience of most general physicians the combination is a rare one, though alienists, on the other hand, regard it as less uncommon. Thus Moebius has not observed a single case of undoubted "Basedow-insanity." As far as I am aware, only three of my 180 cases developed insanity. In one of these there were suicidal tendencies for three months following parturition, so that the condition was possibly puerperal in origin. In another case, that of a man who had melancholia with suicidal tendencies, the insanity appeared to be due to the Graves's disease. In the third case, which I saw with Dr. Swanwick of West Hartlepool. a young lady of 22 had entered her brother's room just after his tonsil had been removed and gaw the excised tonsil lying there, which gave her a shock. She then developed an attack of acute mania, during which ohe was very restless, striking at any one within reach; if left for a minute she jumped out of bed and rushed to the window, used bad language, declined to answer questions, and refused medicine, saying it was poison. She afterwards completely recovered from the mania. Dr. Clouston ${ }^{1}$ at the Edinburgh meeting of the Neurological Society showed three cases of exophthalmic goître who were affected with ideas of persecution and of grandeur, and expressed the opinion that insanity ultimately developed in a much larger number of cases than was generally supposed. Insomnia occurs in a few cases, and headache, especially of a migrainous type, is not uncommon.

A fine regular tremor of the hands is one of the most constant symptoms of exophthalmic goître. The importance of this symptom has only been fully recognized since its great frequency was pointed out by $P$. Marie in France, and its characters were so well described by $\mathrm{Mr}$. A. Maude in this country. This tremor is present in the great majority of cases. This symptom was observed in 163 of my cases, in 7 it was absent, and in the remaining 10 it was not noted. It sometimes appears quite early in the course of the disease, more frequently, however, it does not develop until a later stage, when other symptoms have been present for some little time. The tremor is most easily observed when the arms are bared and fully extended forward with the palms directed downwards. It varies considerably in its amplitude in different cases, and even in the same case at different periods. In the slighter forms it may not attract the attention of the patient at all, whereas in severe cases the patient may be unable to write or carry a plate without dropping it. The movement, which in slight cases is confined to the hands, is due to rapldly alternating contractions of the flexors and extensors of the wrists. In more severe cases the pectorales, serratus magnus, and the erector spinae at times also show a tremor. The tremor is often present in the legs as well as in the arms, and is generally symmetrical, though it is sometimes more marked on one side than the other. The rhothm is rapid and regular. Careful tracings taken by $P$. Marie in 11 cases showed that the movements occurred at the rate of from 8 to 9.5 a second, the average being 8.5. Wolfenden and Williams give the rate of vibration as 10.8 to 11.5 . The amplitude of the tremor varies considerably. It is greater when the patient is excited or when the symptoms are at all acute. Painful cramps may occur in the feet and legs, especially at night, and have been observed by Dr. Hector Mackenzie in 13 cases. In two of these the muscular spasm was similar to that which occurs in tetany. Similar attacks have also been seen by Mr. Maude.
As far as my own experience goes, the cramps are rare symptoms. Convulsions sometimes occur, and may simply be due to epilepgy developing as a complication. In some cases, however, convulsions occur which appear to be the direct result of the exophthalmic goître. I shall have occasion later on to refer to a case in which convulsions occurred while the patient was taking rodagen. Paraparesis, or sudden giving way of the legs, is a symptom first described by Charcot. In some cases the legs simply feel weak as if they would give way in walking, in others the legs actually do give way and the patient falls to the ground; 21 of my patients complained of this symptom, II stated the legs only felt weak, while in 7 the legs gave way and 5 fell down or were unable to walk in consequence; one of them was unable to rise again afterwards without assistance. Other forms of functional paralysis may occur in Graves's disease, but time will not permit of their consideration on this occasion.

\section{SkIN.}

The skin is usually rather pale, though it is sometimes flushed, and it is frequently moist or greasy in appearance as a result of the increased sebaceous secretion. To the touch it is warm and clammy. More or less profuse perspiration occurs at times in many cases. This moist condition of the skin was present in I 33 of my cases, and actual sweating occurred in 92. The sweating may be quite local, being limited to the head, hands, or feet, or one side of the body. Profuse offensive night-sweats were described by von Basedow.

Pigmentation of the skin, in one form or another, was observed in 42 of my cases. In its most common form the pigmentation affects the exposed portions of the skin of the face, neck, and hands, the colour being just like that produced by prolonged exposure to the sun. As a rule the pigmentation is most marked in those parts of the body where the skin is naturally darkest. Thus, the skin round the eyes and covering the eyelids may be quite deeply pigmented while the rest of the face is unaltered. The skin of the face may, however, be uniformly pigmented, or the coloration may occur in dark brown patches on the cheeks. The neck, the axillae, the areolae of the nipples, the genital organs, the upper part of the inner surface of the thigh, and the flexures of the joints, are all common situations in which pigmentation occurs. Portions of skin which are subjected to pressure or friction by clothing are liable to become dark in colour, as for example, over the spines of the vertebrae, round the waist, or below the knee when garters are worn. Variations in the distribution of the pigmentation occur in different cases. In one of my cases the outer edge of the areola and the front of the leg showed the pigmentation most distinctly, while in another there was general diffuse pigmentation of the face, neck, forearms, and hands, with darker, freckle-like patches on the backs of the hands and forearms. The pigmentation may be so general as to resemble that of Addison's disease, or it may occur in small patches like freckles, or in large sharply-defined areas. The mucous membranes are usually unaffected. Leucoderma has been observed in several cases. Various skin rashes have been observed to occur, the most common of which are erythems and urticaria. Two distinct varieties of oedematous swelling may occur. First, ordinary oedema of the lower limbs or elsewhere may develop as a result of heart failure or when the case is complicated by the presence of anaemia, varicose veins, cardiac or renal disease. The oedema is then symmetrical, and pits easily on pressure. Secondly, irregular oedematous swellings of the skin may appear in different parts of the body, such as in the eyelid, on one hand or foot, or elsewhere. In one of my cases a considerable oedema developed in one leg below the knee, for which no local or general cause could be discovered. The diminution of the electrical resistance of the skin has been carefully investigated by several observers, and is simply the result of the increased activity of the sweat glands, in consequence of which a larger amount of moisture is present in the deeper layers of the skin, which is thereby rendered a better conductor. The hair is often profoundly affected. In many cases it becomes ill-nourished and loose, so that it comes out freely. Loss of hair was noted in 29 of my cases. Not only is the hair of the head affected, but that in other parts of the body may also be lost. In 2 of my cases there was complete baldness, and in one of these the eyebrows, eyelashes, and some of the pubic hair had also been lost. The hair may turn rapidly grey, and Mr. Maude quotes a case in which it turned white in a few days. 
Thi Alimentary Srgtem.

The alimentary system is apt to be affected in varions ways. The appetite varina in different cases and at different stages of the disease. During the earlier stages of the malady the appetite is frequently increased. In some cases it is even voracious, and there is a constant craving for food, of which large quantities are consumed. Some alteration in the appetite was noticed in 20 of $\mathrm{my}$ cases. In 4 it was diminished, and in 16 it was increased or voracious. In ro others it was stated to be good. Thirst was only complained of in 7 cases.

Vomiting may occur from time to time, commencing and ceasing again suddenly, independently of any kind of food. These "gastric crises" are generally painless, and watery mucus alone is brought up which may be streaked with blood. In one of von Graefe's cases vomiting occurred from ten to twenty times a day for a month. Dr. Dreschfeld in 7 cases in which these attacks occurred observed that the breath had a peculiar sweet odour, while the urine smelled of acetone and gave the diacetic acid reaction. Vomiting should always be regarded as a serious symptom, to be checked as soon as possible, as in some cases it comes on acutely and persists until the patient dies from exhaustion. Vomiting occurred in 11 of my cases. In 3 of these it was severe, and continued until death.

An increased frequency of defaecation was present in 20 cases. Thus patients who in health were wont to pags a single daily motion defaecated twice or thrice in the twentyfour hours. This symptom must be carefally distinguished from diarrhoea, as the motlons, though more frequent, are otherwise normal in character. The most common disorder of the alimentary canal, however, is diarrhoea, which generally occurs in sudden, short, painless attacks of one, two, or three days' duration, coming on without apparent cause. Some three or four light-coloured w'atery motions are passed in the twenty-four hours, while the tongue remains clean, and there is little constitutional disturbance. The attacks may recur from time to time. They sometimes are very severe; thus in one of my cases from twelve to eighteen motions were passed in the twenty-four hours, and some cases have been recorded in which thirty or forty motions were passed in the day. Diarrhoea occurred in 53 , or more than one-quarter of my cases. Constipation was present in 16 .

Albumen is occasionally found in the urine in small quantities. It is usually intermittent, and the urine contains no casts. In 30 of my cases in which the urine was examined for albumen a trace was found in 6 . In only 3 of these was sugar found to be present, in 1 of them the urine contained 15 grains of sugar and in another 40 grains to the ounce. As a rule this glycosuria may be distinguished from diabetes by the fact that it does not progress but tends to disappear as the patient improves. I have, however, seen a case recently, which is not included in this series, in which diabetes with thirst, wasting and other symptoms occurred as a complication. In the large majority of cases the urine is quite normal.

In a considerable number of women who suffer from exophthalmic goitre menstruation is abnormal. In 170 cases in women menstruation was affected in 45. Amenorrhoea, whlch is not uncommonly present during the earlier stages of the malady, existed in twenty-lour of my cases for periods varging from five months to elght years. In several of them menstruation returned as the other symptoms of the malady abated. In the other cases the variations in menstruation were either unimportant or were accounted for by some local cause. In a few cases the disease has first developed during pregnancy. Maude quates two cases in which this occurred; in each of them the placenta becamedetached and the fetus died. In one case the symptoms rapidly subsided after delivery. Pregnancy may also occur in a woman who is already snffering from exophthalmic goître. When this does occur it is important to remember that marked improvement may take place during the pregnancy, as in three cases observed by Charcot, and that'it should therefore be allowed to proceed to a natural termination.

Nutrition varies considerably in different cases, hut some diminution in weight occurs in many cases. Loss of weight may take place during the early stages of the disease, even in spite of the appetite being increased and the amount of food consumed larger than usual. Some loss of weight was noted in 67 , or rather more than one-third, of my cases. The actual loss varied considerably in different cases, but amounted in some to as much as 3 st. or $4 \mathrm{st}$. In one male case the patient when first seen had a simple parenchymatons goitre. When examined again, two and half years later, he was found to be suffering from a severe form of exophthalmic goitre, and had lost from $2 \frac{1}{2}$ st. to 3 st. in weight. A regain of weight is a favourable sign of general improvement. Thus one of my patients weighed only 6 st. when the disease was fully developed. When she was seen again, eight years afterwards, she weighed 9 st. I I lb., a gain of nearly 4 st., and the other symptoms had subsided.

Treatment.

The treatment of exophthalmic goitre is a matter of considerable difficulty, 28 it is a much more formidable task to control the excessive secretory activity of the thyroid gland than to supplement its deficiency, as in the treatment of myxoedema and cretinism. In estimating the value of any particular line of treatment in Graves's disease we have to remember how variable the natural course of the disease may be in different cases, so that it is no easy matter to determine in any given case whether an improvement in the patient's condition is really due to the treatment adopted or is merely incidental to the natural couree of the malady. In spite of this difficulty, however, we can, I think, in the majority of cases, with the means which are now at our disposal, not only relieve special symptoms as they arise, but also favourably modify the course of the disease as a whole.

In dealing with this part of my subject I propose to consider separately the general hygienic treatment, the influence and uses of various drugs, the electrical treatment, and, finally, the most recent results obtained by the use of antitoxic serums and rodagen.

The general management of the patient is of great importance, and has, of course, to be modified according to her character and social position, and the stage of the disease. In cases of great severity, and in less severe cases during the exacerbations which occur from time to time, complete rest in bed is essential. This may be continued for periods varying from a fortnight to six weeks, according to the progress made. In cases of moderate severity a rest in bed for two or three weeks at the commencement of a course of treatment is advisable. In slight cases treatment in bed is not required. In severe cases in which rapid loss of weight has occurred the rest in bed should be combined with over-feeding, as much as four or five pints of milk being given in the twenty-four hours in addition to the regular meals. Little difficulty is usually experienced in inducing such patients to take a large amount of food, as they not infrequently have a voracious appetite. Strict isolation, as in Dr. Weir Mitchell's method of treatment, is not, however, required. After this period of rest, or in less severe cases from the beginning, it is important as far as possible to regulate the patient's mode of life. As a considerable amount of rest is very important, it is a useful plan to insist on at least twelve hourg' rest in bed, say from 10 p.m. to 10 a.m. In addition to this the patient should lie down for an hour or two during the day. As much time should be spent in the open air as possible. The combination of rest with open-air, as in the treatment of pulmonary tuberculosis, gives excellent results.

Without giving further details, it will suffice to say that the patient should be induced to lead as quiet and easy an outdoor life as she can, freed as much as is possible from anxiety, from undue mental effort, from uncongenial social duties and from unsuitable physical exertion.

\section{Electricity.}

One of the most valuable means of treatment of Graves's disease is undoubtedly the systematic application of a faradic current. This treatment, which may be employed alone or along with medicinal treatment, should be carried out wherever possible, as in my cases the best results have been obtained with patients who have persevered with it. The most satisfactory method of applying the current is that suggested by Sir Victor Horsley. Two flexible electrodes about 4 in. long and 2 in. wide, covered with flannel or washleather are moistened with a warm salt solution one is applied in front over the goître and the other at the back of the neck. These electrodes are fitted with straps and buckles. at the sides, by means of which they are fixed in position. They are then connected with the secundary circuit of a drycell faradic battery, a water rheostat being included in the circuit, so that the strength of the current can be regalated as desired. The current should be just strong enough to produce a prickling sensation in the skin, bat not 80 strong as to be anpleasant. The current should be applied in this manner for an hour twice in the day, and in some cases it 
may be applied for as much as three or four hours in the day. A patient very quickly learns how to apply the current herself. This treatment can be carried out easily in the case of private patients and in a hospital, but it is unfortunately not adapted for hospital outpatient practice, in which so many of these cases are seen The drawbacks are the time and trouble it entails, so that steady perseverance on the part of the patient is essential. I have observed steady improvement and practical recovery take place in several cases in which this treatment has been systematically carried out.

$X$ Rays.

The remarkable effect $x$ rays have been found to possess in bringing about a reduction in the size of an enlarged spleen in cases of spleno-medullary leukaemia has suggested that they might be of service in the treatment of exophthalmic goitre. In one of my cases in which this treatment was adopted no benefit was derived; indeed the symptoms were somewhat aggravated, and so it was discontinued. Beck, after excision of one lobe of the enlarged thyroid, exposed the other lobe to $x$ rays. In two cases in which this treatment was carried out-in one eighteen, and in the other three, months after the operation, which had only produced an improvement-a remarkable diminution in the nervousness and tachycardia was observed. In a third severe case, in which the $x$-ray treatment was started a week after the operation by a daily exposure of at first five and then ten minutes, the improvement was immediate and rapid. At the end of a week the treatment was suspended for a week, when it was found the pulse had fallen from 160 or 180 before the operation to 80 , and the exophthalmos had nearly disappeared. After that the $x$-ray treatment was given for five minutes each week. When seen five months after the operation the remaining balf of the goitre had disappeared, as had also the exophthalmos and tachycardia. It is, however, difficult to determine whether this satisfactory result is to be attributed to the operation or to the $x$ rays, or to both. Dr. Stegman ${ }^{3}$ treated two cases of Graves's disease by $x$ rays with marked success, each sitting occupying fifteen minutes. The nervous and cardiac symptom disappeared, though the size of the goitre remained much the esme. These results suggest that a further trial of $x$-ray treatment should be made.

In a large number of cases, and especially those which are treated in out-patient departments, the treatment is practically confined to the administration of drags, so that it is important to consider what influence they exert on the course of the disease. Fortunately even under adverse circumstances on sees some cases do well on medicinal treatment alone. The number of drugs which have been recommended in the treatment of this malady is large, but as far as my own experience goes I have only found a few to be of real service.

Arsenic is one of the most useful for routine treatment, small doses being given. A useful method is to order 4 or 5 minims of liquor arsenicalis to be taken three times a day, except during one week each month or during the menstrual period, for six, eight, or even twelve months. When the pulse-rate is over 110 , 10 or 15 minims of tincture of convallaria is added to the dose, as this drug appears to give better results than either digitalis or strophanthus in controlling the excessive pulse-rate. If nervousness is a marked feature in the case, $10 \mathrm{gr}$. of potassium bromide may also be added to the mixture for a few weeks at a time. Belladonna is useful in some cases, and may have some influence in checking the hypersecretion of the thyroid gland; of this, however, we have no actual proof. It may be used in cases where excessive perspiration is a source of annoyance, but is not suited for prolonged administration. Iodide of potassium is, I consider, quite unsuitable, as it not infrequently aggravates the symptoms; and this is not surprising when we remember that thyroid secretion, which contains iodine in an organic combination, is being poured into the blood in excessive quantities; for the same reason thyroid extract, which is still unfortunately given in some cases, has not infrequently aggravated the symptoms, and should therefore never be given. In some cases in which thyroid extract had been prescribed, I have seen rapid improvement take place as soon as it was discontinut d.

Suprarenal and thymus tablets have been of service in some cases. Sodium phosphate in doses of $15 \mathrm{gr}$. to $20 \mathrm{gr}$. which has given good results in the hands of some observers has not appeared to me to produce any marked beneficial effect. The daily application of a red iodide of mercury ointment, of half the usual strength, over the goitre has appeared to be useful in many of my cases.
Special measures are not infrequently required in the treatment of urgent symptoms. The sudden attacks of diarrhoea, to which some patients are so liable, can be controlled by rest, liquid diet, and a mixture containing laudanum and dilute sulphuric acid. Persistent vomiting may be at times a formidable symptom, and in several of my cases it has been uncontrollable, causing death from exhaustion. In these cases morphine, given either by hypodermic injection or in a suppository, has proved more efficient than any other drug. Severe attacks of palpitation, in which the pulse was 180 to 200 , yielded to the application of an ice-bag to the praecordium.

During the last few years the problem of the treatment of exophthalmic goître has been approached in a more rational manner, based on our increasing though still very imperfect knowledge of the part played by the thyroid gland in the production of the symptoms already referred to. Endeavours have been made on several different lines to neutralize the harmfal $\mathrm{effects}$ of the excess of thyroid secretion present in the blood. In the earlier experiments it was assumed that in health the function of the thyroid secretion is to neutralize or destroy certain products of metabolism present in the blood or tissues. When the thyroid secretion is lacking, either as the result of disease or removal of the gland, these substances were supposed to accumulate in excess in the blood. It was considered that this blood containing these bodies might conversely possess the power of neutralizing the excess of thyroid secretion present in the blood in Graves's disease. Proceeding on these lines, Ballet and Enriquez, in 1895, first employed the serum of dogs, from which the thyroid gland had previously been removed, and in which symptoms of athyroidism had appeared, in the treatment of exophthalmic goitre. They injected from 4.5 to $15 \mathrm{cccm}$. of the gerum with beneficial effects: Following up this same idea, Burghart and Blumenthal ${ }^{5}$ considered that the blood of a patient suffering from myxoedema would have a similar antitoxic action when employed in the treatment of exophthalmic goitre. For this purpose they removed $200 \mathrm{c.cm}$. of blood from $a$ fully-developed case of myzoedema. The blood was mixed with an equal quantity of normal saline solution and chloroform and placed in the refrigerator for twenty-four hours. It was then filtered, first through liner then through a Berkefeld filter, and after the addition of more chloroform replaced in the refrigerator. This fluid was injected into a girl, aged 15, who was suffering from a severe form of Graves's disease, in doses varying from 20 to $55 \mathrm{c.cm}$., generally of $25 \mathrm{c.cm}$, the total amount injected amounting to 247 c.cm. in eight weeks. During this treatment the patient gained weight, the hair grew again, the exophthalmos diminished, the sweating and tremors were less, and the heart was quieter and more regular in action. The symptoms were, however, reported to have returned after the patient left the hospital.

Burghart and Blumenthal also made use of the blood of thyroidless dogs. For this purpose they removed the thyroid from eight dogs, two of which remained well, and six developed tetany. These six were bled from the femoral artery. At first the blood was prepared as already described, later the dried precipitate thrown down by alcohol was ased instead. The liquid was injected in doses varying from 15 to $25 \mathrm{c.cm}$. The dried serum, one gram of which was found to be equivalent to $10 \mathrm{c.cm}$. of the liquid, was given by the mouth in doses of 1 to 1.5 grams. Considerable improvement was observed in each of the cases treated.

Moebius, ${ }^{6}$ working in the same direction, employed serum prepared from the blood taken from sheep several weeks after thyroidectomy, and reported favourably upon its effect given in 5 minim doses three times a day. This serum, as prepared by Merck, is now to be obtained under the name of antithyroidin.

Schultes ${ }^{7}$ treated a very severe case of exophthalmic goître, with acute mental symptoms, with this serum with remarkable results. After a month's treatment on doses varying from 0.5 to $45 \mathrm{cccm}$. thrice daily, the mental symptoms had entirely disappeared. the pulse-rate decreased from 140 to between 80 and 90. The circumference of the neck decreased from $44 \mathrm{~cm}$. to $42 \mathrm{~cm}$. The tremor diminished 80 that the patient could again thread a needle. The exophthalmos was unaffected; except for this the patient appeared to have made a good recovery in a very short time. The after-history is not given. P. Sainton and $B$. Pisante ${ }^{s}$ treated three cases, first with the glycerinated blood, and later with the serum of a thyroidless sheep. In one case the result was considered to be 
a complete and permanent cure, and in the other two marked improvement took place. Attention was specially drawn by these observers to the favourable influence of the treatment upon the tachycardia as evidence of its efficiency; thus in the first case the pulse decreased from 140 to 80 , in the second from 125 to 90 , and in the third from 130 to 80 . Preference was given by them to the oral administration of the serum, which proved to be more active than the glycerinated blood. All three cases had previously resisted other medicinal treatment. the first having been treated by thymus extract, the second by electricity, and the third had been in a stacionary condition for three years.

Leyden, ${ }^{9}$ who has employed Moebius's serum, rodagen, and Madsen's tablets of dried blood from a thyroidless goat, was unable to decide that any one of them was decidedly more efficient than the others, though he appears to have obtained good results with rodagen, which will be referred to again.

My own experience of Moebius's serum is very limited, but I have not been able to observe any special effect in those cases in which I have employed it, and Dr. Hector Mackenzie after an extended trial came to the same conclusion. O "thyroidectin," a new preparation of the blood of animals after thyroidectomy, I have as yet no practical experience.

Lanz, working on similar lines to Moebius, considered that the milk of an animal from which the thyroid gland had previously been removed would contain the same substances as the serum, and might therefore be used instead. Lanz obtained good results in the treatment of six cases by this method. The best method of carrying out this treatment appears to be to employ the fresh milk from a thyroidless goat, the whole of the milk of one goat being consumed by the patient in the twenty-four hours. When the fresh milk cannot be obtained, the dried milk may be employed instead. and is sold under the name of rodagen. W. Kuhnemann ${ }^{10}$ has recorded a case of well-marked exophthalmic goître, in which, after other treatment had failed, great improvement took place while taking $\frac{1}{3}$ drachm of rodagen three times a day. In the course of five or $81 x$ weeks' treatment the circumference of the neck diminished from $35^{\frac{1}{2}}$ to $34 \mathrm{~cm}$. The pulse, which before treatment was between 135 and 145 , diminished to 90 . The weight increased from 97 to $109 \mathrm{lb}$. The patient was able to walk better uphill without dyspnoea. An apical systolic murmur and the tremors disappeared. When the rodagen was discontinued the pulse within a few days increased to between 106 and 120 , and the weight decreased. On resuming the rodagen, improvement again took place and was maintained after it was discontinued.

I have not employed the fresh milk of a thyruidless goat, but shall now shortly refer to one of my cases which was treated by rodagen. The patient was a young woman, aged 22, who had first developed symptoms of exophthalmic goître some twelve months before her admission to the Newcastle Royal Infirmary on January I6th, 1905. She had well-marked exophthalmos, considerable enlargement of the thyroid gland; the pulse was 140, and she had fine tremors of the hands, and was in fact a typical example of Graves's disease. She remained in the hospital until the end of March. She was given I drachm of rodagen thrice daily from January 18th for one week only, as the supply failed; again from February 3 rd to February 24th, and again in $\frac{1}{2}$-drachm doses from March 8th to April 2nd, so that altogether she took 15 ounces of rodagen while she was under treatment. For about one week, during which the rodagen could not be obtained, she took I drachm of sodium phosphate each morning instead. After she began to take the rodagen a steady improvement took place. The pulse, which at first varied between 100 and 140, fell to between 96 and 116 . The tremors diminished. On February 2oth the temperature rose from normal to $1038^{\circ}$, and she developed an acute tonsillitis. On February 24th, that is after she had taken the rodagen continuously for three weeks, she became pale, and collapsed at 7.30 a.m.; the temperature, which the night before had been $102^{\circ}$, fell to $99^{\circ}$. At noon the temperature was only $95.2^{\circ}$, and the heart-beat, which during the four previous days had been accelerated to between 120 and 160 , fell to 32 per minute. The skin was cold and moist. She was somewhat cyanosed, and a systolic bruit could be heard at the apex. The rodagen was discontinued, and brandy and strychnine were given. The pulse at 2 p.m. was 36 ; at 4.44 ; and at 11.30 p.m., 96 . The following day the patient appeared to be in her usual condition, but the pulse was only 88. Two days later, on February 27 th, the pulse again fell at 6 a.m. to 76 , at 7.20 to 64 , at 8 to 44 , at 8.30 to 42 . At
10.15 it was only 38. After this it gradually increased in Irequency, and was 62 at $11.45,52$ at 1.45 p.m., 46 at 4,88 at 8 , and 96 at 11 p.m. During the morning of this day, between 7.20 and il a.m., she had numerous attacks, in each of which the heart, which was as already described beating slowiy, would stop altogether for several seconds; a convulsion then took place, which lasted two or three seconds. The patient was conscious of the onset of the convulsion, but lost consciousness for a second or two at the end of it. One or two of these attacks occurred while I was auscultating the heart, and the long period of asystole was remarkable; the slow pulse was irregular at the time. After I a.m. she had no return of the attacks. During the month of March the pulse varied from 64 to 114, but was only as high as 100 on ten days. Rodagen was again given in $\frac{1}{2}$ drachm doses thrice daily on March 8th, and continued until she left the hospital on April 2nd.

I have ventured to detail this case as I have never seen symptoms like these before in a case of exophthalmic goitre, and am, therefore, inclined to attribute them to the action of the rodagen in too large doses. As no untoward symptoms occurred when $\frac{1}{3}$.drachm doses only were given later on, and the patient left the hospital very considerably improved, I think this remedy is worthy of more extended trial, but it should be used with caution in doses not exceeding $\frac{1}{2}$ drachm.

As the symptoms of exophthalmic goître are due to the overactivity of the thyroid gland, and improvement takes place when atrophic changes occur in the gland, it would be a rational means of treatment to endeavour to induce such degenerative changes in the secreting cells of the gland by means of specific cytolysins, and so to lessen its activity. Thus Mankovsky ${ }^{11}$ introduced the thyroid glands of dogs into the peritoneal cavity of cats at intervals of a fortnight. After three injections he injected the cat's serum into healthy dogs. This was followed by symptoms like those which develop after thyroidectomy and by degeneration of the epithelium of the gland. Goutscharukov, ${ }^{12}$ by injecting dog's thyroid into rams, prepared a sheep's serum which he considered to be thyrotoxic, as its use was followed by tetany associated with loss of colloid, vacuolarization, and chromatolysis of the cells of the thyroid. Portis ${ }^{13}$ also prepared a serum by injecting increasing doses of an emulsion of dog's thyroid into the peritoneal cavity of the goat. When the goat's serum was subsequently injected into dogs it produced depression, convulsions, vomiting, rapid breathing, haemoglobinuria, and early death. In cases which survived longer there were some fever, lachrymation, and progressive loss of welght. Microscopical examination of the thproid gland showed removal of colloid, desquamation and degeneration of the epithelial cells, followed later by the development of papillary prolifera. tions. Marked degenerative changes were, however, found in the liver, spleen, and kidneys, so that the action of this serum is not solely thyrotoxic. It would, therefore, be unsuitable for the treatment of exophthalmic goître, us degenerative changes would be set up in other organs as well as in the thproid gland. MacCallum, and later J. L. Yates, saw no results follow injections of a thyroid serum prepared for the dog in geese.

It has appeared to me that by working on somewhat similar lines it might be possible to induce the formation of antibodies in the blood, the gerum of which might be employed in the treatment of exophthalmic goitre, so as to neutralize the ill effects of the excess of thyroidal secretion. It has been shown by several observers that not only serums which are antitoxic to certain bacterial poisons, like the anti-diphtheritic serum, but also serums antagonistic to animal products, can be produced. Thus, Lepine ${ }^{15}$ endeavoured to prepare an antithyroid serum by first injecting thyroid extract into a goat, later feeding the animal on graduallyincreasing doses of minced thyrold until it was able to take as much as twenty to twenty-five lobes of the sheep's thyroid a day. The serum prepared from the goat, when injected into a dog, caused diminution in the excretion of urea, and in large doses somnolence and slowing of the pulse, which was taken as an indication of interference with the activity of the thyroid gland. This serum, however, has apparently not been employed in the treatment of exophthalmic goitre.

In my own experiments I endeavoured to prepare an antithyroid serum in as simple a manner as possible-first of all from rabbits, ${ }^{16}$ and more recently from a goat. In the first experiments several rabbits were fed on sheep's thyroid extract for a month or more. Each rabbit was killed by bleeding, and the blood was collected with aseptic precautions 
into sterilized flasks. After standing for forty-eight hours the serum was decanted, and 0.2 per cent. of carbolic acid added to it. Two cases of exophthalmic goître were treated with the serum in doses varying from 5 minims once a day to 7 minims three times a day. Both cases improved under ureatment, but the amount of improvement was not greater than might be expected from rest in the hospital and ordinary medicinal treatment, and I was unable to attribute any specific effect to the use of this serum.

More recently I prepared a serum in a similar manner from a goat. In this case, however, the period of preparation was much longer. A young she-goat, nine months old, was selected. Sheep's thyroid extract, the liquor thyroidei (B.P.), was employed throughout. This was at first injected beneath the skin, and from March 9th to April 26th 13 injections were made, the doses ranging from $06 \mathrm{c} \mathrm{cm}$. to $5 \mathrm{c.cm}$., $27.5 \mathrm{c.cm}$ being injected altogether. As localized abscesses developed after several of the injections, the method of administration was changed, and for a period of two months, from May 17 th to July 17 th, the extract was given by the mouth in doses varying from $2 \mathrm{c.cm}$. to $8 \mathrm{c.cm}$. In all during the period $86 \mathrm{c.cm}$. were given in 22 doses, which thus averaged about $4 \mathrm{c.cm}$., or $1 \mathrm{dr}$. each. On July 24th 400 c.om. of blood were withdrawn from the internal jugular vein by means of a curved hollow needle into a sterilized flask, while the goat was under the influence of an anaesthetic. After coagulation had taken place $170 \mathrm{c.cm}$. of clear serum were decanted into sterilized bottles, 0.5 per cent. of carbolic acid being added to it. Two cases have been treated with this serum.

The first case was that of a single woman, aged 22, who, after being much frightened by a dog, developed symptoms of exophthalmic goître, some six weeks before her admission to the Newcastle Royal Infirmary. At this time she had very marked exophthalmos associated with retraction of the upper eyelids and von Graefe's sign. The thyroid gland was moderately enlarged. The pulse was 120, regular, and of medium tension. Systolic murmurs were audible at the apex and over the pulmonary area, otherwise the heart was normsl. The skin was moist and pigmented in places. There was a marked fine tremor of the extended hands, and she was nervous and restless, so that the case was a good typical example of the disease.

Treatment was commenced on July 26th, by first giving 5 minims of the serum three times $a$ day. The dose was increased to 7 minims on the 28 th, and to 10 minims on August Ist. The pulse then had gradually decreased to 100, the nervousness and restlessness had diminished, and the goitre appeared to be getting smaller. On August 5 th the dose was increased to 20 minims, that is I drachm in the day, and with the exception of two days continued up to August 19th. During this time the pulse varied between 106 and 120, and the exophthalmos became slightly less marked. The weight remained practically unaltered. In this case, although some improvement took place, it was not more than usually takes place in hospital on ordinary treatment, and no special effect could be traced to the use of the serum.

The second case was that of a young woman, aged 23 , in whom the symptoms of exophthalmic goître had first deveveloped about four months before she came to the Royal Infirmary on July 22nd. On admission she had slight uniform enlargement of the thyroid gland. The exophthalmos was slight, and the ocular symptoms were absent. The pulse was 126, small, regular and easily compressible. The heart's apex beat was in the filth intercostal space, one fingerbreadth outside the nipple-line. A rough systolic murmur was audible over the pulmonary area, but not at the apex. The skin was moist and pigmented, and there were other minor symptoms which I need not detail. She was given 5 minims of the goat's serum three times a day on July $26 \mathrm{th}$, the dose being gradually increased up to 20 minims by August 5th. This dose, with the exception of two days, was continued up till August igth. The pulse varied between 104 and 120 , and there was an increase of $8 \frac{1}{2} \mathrm{lb}$. in weight. The general condition also improved, but, as in the other case, no special effect could be attributed to the use of the serum.

The risks of surgical treatment are unfortunately so great that I do not consider partial thyroidectomy is advisable in exophthalmic goître. I have only seen this operation performed in two cases, but they both died within an hour or two. We must, however, remember that Kocher's results in 59 cases, quoted by Dr. Mackenzie, ${ }^{17}$ show 76 per cent. of cures, and improvement in 14 per cent., with a mortality of only 6.7 per cent. If operative treatment is undertaken a local ansesthetic only should be employed, as much of the risk appears to be due to the use of general anaesthesia.

We must on the whole conclude that at the present time in the great majority of cases the best results are obtained by general hygienic treatment combined with the use of electricity and certain drugs, and that as yet no serum or other animal product can be considered to give better results than these older methods of treatment.

REFERENCES.

1 Brain, 1903, p. 3co. 2 Abstract, La semaine Médicale, July rgth, 1905, p. 340. 5 Dertsch. med. Wh neurologistes franç18 de Bordsaux, August, 1895. No. 22.7 Milinch. med. Woch. Mav, 1899, p. 627. 8 Revue Neurologigue, No. 22 . Minch. med. Woch., Map. rgoz, p. 834.

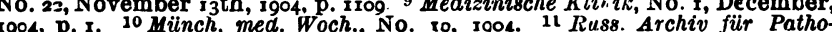
1904, p. x. 10 Minch. med. Woch., No. ro, ro9. It Rus8. Archiv fir PathoAnat., Tog. is Journal of Infectious Diseases, No. J, January 2 nd, 1904. November 2 th 1903 . 16 Lancet, August 27th, 1904. 17 BRITISH MEDICAI JOURNAI, October 28 th, 1905 , p. 1082.

\section{A CONCEPTION OF DISEASE.}

Being the Inaugural Address delivered before the Philosophicat Instutution of Edinburgh.

By Sir FREDERICK TREVES, BART., G.C.V.O., C.B., LL.D., Serjeant-Surgeon to H.M. the King.

Among the items which have in recent years been considered necessary to a liberal education is some knowledge of the human body, of its mechanism and incidentally of its infirmities. Physiology is taught in schools, demonstrations of "first aid to the injured" and classes in ambulance work are popular throughout the country, the elements of hygiene are matters of common knowledge, a very general interest is taken in public health, and especially in preventive medicine, while there are few who have not some conception of germs and of the part they play in the troubles of life.

The result is an intelligent concern in medicine which is of infinite advantage to the whole community. There has grown up a kind of popular medicine, founded, the cynic would say, upon that "little knowledge which is a dangerous thing," but based, I would rather insist, upon that little knowledge which suffices to teach the indications of danger. The foundation of any system of medicine is a right appreciation of disease. I venture to think that the conception of disease which is the basis of medicine $a$ la mode is not in accord with facts, and, influenced by this impression. I would attempt to present a religio medici which, I trust, rests upon surer grounds.

The prevailing idea of disease is of a something that is woful and malignant, evil in origin, evil in intent, evil in effect. The method of its entering into the lives of men would seem to belong to the time

Of man's first disobedience and the fruit

Of that forbidden tree, whose mortal taste

Brought death into the world, and all our woe.

The popular view claims that disease is a calamity, that its end is destruction, and that it is purposeless except in the direction of doing harm. Popular terms bear testimony to the prevailing of this belief. Man is aaid to be struck down by disease as by an avenging angel, it seizes upon him as does a roaring lion, it consumes him as does a fire. The attitude of the medical man towards disease is that of an opponent to deadly influences. He has to combat an enemy to mankind whose every wovement is dark and malicions. There is no symptom of disease that is not believed to be noxious, and as such must be stamped out with relentless determination. If the patient be sick, the sickness must be stayed; if he cough, the cough must cease; if he fail to take food, he must be made to eat. And why? Because these are manifestations of disease, and are therefore of ill.intent and need to be banished.

I ghall hope to show-so far, at least, as the examples I shall select are concerned - that there is nothing preternatural in disease, that its phenomena or symptoms are marked by a purpose, and that that purpose is beneficent. I shall hope in these examples to demonstrate that the processes of disfase are aimed not at the destruction of life but at the saving of it, and that its manifestations are the outcome of a natural effort towards cure.

Disease, as popularly realized, is not one of the ills that flesh is heir to, but one of the good gifts, for its motive is benevolent and protective. I cannot express this more 